\title{
Nonequilibrium Magnetization Dynamics of Gadolinium Studied by Magnetic Linear Dichroism in Time-Resolved $4 f$ Core-Level Photoemission
}

\author{
A. Melnikov, ${ }^{1}$ H. Prima-Garcia, ${ }^{2}$ M. Lisowski, ${ }^{1, *}$ T. Gießel,,${ }^{2, \dagger}$ R. Weber, ${ }^{2, \ddagger}$ R. Schmidt, ${ }^{2}$ C. Gahl, ${ }^{2}$ N. M. Bulgakova, ${ }^{3}$ \\ U. Bovensiepen, ${ }^{1, \S}$ and M. Weinelt ${ }^{1,2, \|}$ \\ ${ }^{1}$ Freie Universität Berlin, Fachbereich Physik, Arnimallee 14, 14195 Berlin, Germany \\ ${ }^{2}$ Max-Born-Institut, Max-Born-Straße 2 A, 12489 Berlin, Germany \\ ${ }^{3}$ Institute of Thermophysics SB RAS, 1 Lavrentyev Avenue, 630090 Novosibirsk, Russia \\ (Received 26 June 2007; published 13 March 2008)
}

\begin{abstract}
The magnetic linear dichroism of the gadolinium $4 f$ core level is studied in a time-resolved photoemission experiment employing laser pump- and synchrotron-radiation probe pulses. Upon optical excitation of the $5 d 6 s$ valence electrons with femtosecond laser pulses, the magnetic order in the $4 f$ spin system is reduced. Remarkably, the linear dichroism remains at $80 \%$ of the equilibrium contrast while the lattice temperature reaches the Curie temperature due to electron-phonon scattering. Contrasting itinerant ferromagnets, this shows that equilibration between the lattice and spin subsystems takes in Gd about $80 \mathrm{ps}$ and is established in parallel with heat diffusion.
\end{abstract}

DOI: 10.1103/PhysRevLett.100.107202

In current technical implementations changes in the magnetization of ferromagnetic materials are induced thermally, by magnetic fields or by combining both stimuli. While this conventional switching happens on nanosecond time scales, various magneto-optical experiments corroborated in the past decade that the spin system of itinerant ferromagnets exhibits a reduced magnetization within a few hundred femtoseconds (fs) after laser excitation [15]. Unifying concepts have been put forward, but the associated microscopic processes are still notional $[6,7]$. To describe the latter, we have to consider the coupling of charge, lattice, and spin degrees of freedom indicated in Fig. 1(a). For absorbed fluences $F_{a b s}=1-10 \mathrm{~mJ} / \mathrm{cm}^{2}$ typically used in optical demagnetization, a significant fraction of valence electrons is excited and their distribution is no longer in thermal equilibrium [8]. Agreement has been obtained that the initially hot electrons equilibrate with the lattice through electron-phonon scattering as described by the two-temperature model [9]. The outcome of such a simulation for a $100 \AA$ thick Gd film on tungsten is shown in Fig. 1(b) for $100 \mathrm{fs,} 800 \mathrm{~nm}$ laser pulses, and $F_{a b s}=3.5 \mathrm{~mJ} / \mathrm{cm}^{2}$. For $\mathrm{Gd}$ and $\mathrm{W}$ we use the known material constants (see Sec. 3.2 in [10]; [11]); the numerics is described in Ref. [12]. Equilibrium is reached after $1.5 \mathrm{ps}$, when the temperature of electron and phonon subsystems $\left(T_{e}\right.$ and $\left.T_{l}\right)$ are aligned. Concerning the spin subsystem the situation is less clear. Early publications suggest a direct interaction between electrons and spins [1,2], whereas more recent studies favor a phonon- or defectmediated spin flip of excited electrons [6,7]. The underlying argument is based on angular momentum conservation. It requires that a loss of magnetization is compensated, e.g., via spin-orbit coupling [13]. All of these studies explored $3 d$ transition-metal ferromagnets where the magnetic moment is generated by direct exchange interaction among delocalized spins near the Fermi level.
PACS numbers: 75.40.Gb, 75.70.Ak, 78.47.-p, 79.60.-i

By contrast, Gd is a prototype system for a Heisenberg ferromagnet. The magnetic moment per atom of $\mu=$ $7.55 \mu_{B}$ arises mainly from the half-filled $4 f$ shell contributing $\mu_{4 f}=7.0 \mu_{B}$, localized at the ion core. Ferromagnetic order in Gd occurs by indirect exchange interaction (RKKY) below the Curie temperature of $T_{C} \simeq$ $293 \mathrm{~K}$ [14]. Alignment of neighboring $4 f$ magnetic moments is mediated by the spin polarization of the valence electrons generated in turn by the $4 f$ electrons. The intraatomic $4 f-5 d$ exchange interaction is responsible for this polarization, calculated to $99 \mathrm{meV}$ [15]. This valence-band spin polarization of $\mu_{5 d}=0.55 \mu_{B}$ per atom is probed in magneto-optics [16,17]. Corresponding time-resolved measurements provide evidence for an ultrafast drop upon laser excitation $[10,17,18]$. Thus, it is an obvious question whether $\mu_{4 f}$ follows the spin dynamics of the Gd valence band or $\mu_{4 f}$ and $\mu_{5 d}$ need separate treatment. Recently, Bartelt et al. reported on an element-specific magnetization dynamics in $\mathrm{Fe} / \mathrm{Gd}$ multilayers using $\mathrm{x}$-ray magnetic circular dichroism (MCD). Interestingly,
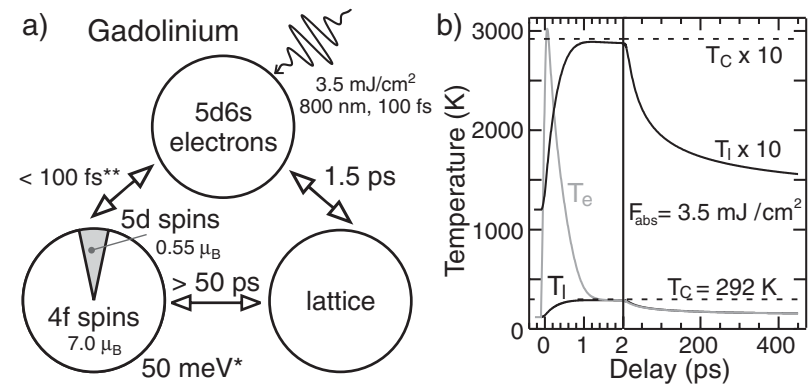

FIG. 1. (a) Energy redistribution among valence electrons, lattice, $4 f$ and $5 d$ spin systems after fs laser excitation, and response times for Gd (* Ref. [15], ** Ref. [17], and this work). (b) Calculated transient temperature of Gd valence electrons $T_{e}$ and lattice $T_{l}$ after laser excitation. 
an almost simultaneous demagnetization is observed probing the Fe valence and $\mathrm{Gd} 4 f$ shell, which could occur, e.g., due to hybridization of both constituents or the intense excitation by $F_{a b s}=20 \mathrm{~mJ} / \mathrm{cm}^{2}$ [19].

In this Letter we report on a combined fs-lasersynchrotron pump-probe experiment. We measure the temporal evolution of $\mu_{4 f}$ directly employing magnetic linear dichroism (MLD) of the Gd $4 f$ photoemission line after Krupin [20]. Under excitation conditions where the lattice temperature $T_{l}$ just reaches $T_{C}$, the magnetization is lowered but remains finite at about $80 \%$ of the equilibrium value. We show that even $50 \mathrm{ps}$ after optical excitation the magnetic moment $\mu_{4 f}$ has not yet equilibrated with the electron and phonon subsystems.

The experiment was carried out at the undulator beam line U125 PGM-1 of the Berlin synchrotron facility (BESSY) operating in single-bunch mode with a repetition rate of $1.25 \mathrm{MHz}$ and a pulse duration of about $50 \mathrm{ps}$. Valence electrons are optically excited by amplified fslaser pulses (800 nm, $100 \mathrm{fs,} \mathrm{RegA,} \mathrm{Coherent).} \mathrm{The} \mathrm{cavity}$ length of the homebuilt Ti:sapphire seed laser is stabilized in order to phase match its repetition rate of $83.3 \mathrm{MHz}$ to the sixth part of the $500 \mathrm{MHz}$ radio frequency cavity of the storage ring. A phase shifter allows one to electronically adjust the delay between laser pump- and synchrotronradiation probe pulses. Injection of seed pulses into the RegA and ejection of amplified pulses are triggered by the BESSY single bunch marker, so that the RegA delivers pulses at a repetition rate of $208.3 \mathrm{kHz}$ phase locked to every sixth synchrotron pulse. Photoelectrons from every sixth synchrotron pulse are selected by an electronic gate set in a time-of-flight multiprobe scheme and are detected after a hemispherical electron analyzer. The overall jitter is below 10 ps [21]. Epitaxial Gd(0001) films of $100 \AA$ thickness, with the easy axis in plane, have been grown on W(110) [10].

Figure 2(a) shows Gd $4 f$ core-level spectra recorded at a photon energy of $60 \mathrm{eV}$ and for a sample temperature of $120 \mathrm{~K}$. The measurement geometry is sketched in the inset. The Gd film was remanently magnetized applying a magnetic field of $0.5 \mathrm{kOe}$. Direction of electron emission $k$, inplane magnetization $M$, and electric field of the synchrotron light $E_{\mathrm{SR}}$ define a chirality, a prerequisite to observe MLD. The $4 f$ spectral shape and intensity $I \uparrow$ and $I \downarrow$ depend on the magnetization directions $M \uparrow$ and $M \downarrow$ [20]. Photoelectrons are detected off-normal $(n)$ at an angle of $(4 \pm 1)^{\circ}$ where the MLD contrast is maximum. The asymmetry $(I \uparrow-I \downarrow) /(I \uparrow+I \downarrow)$ depicted in Fig. 2(b) reveals a contrast as pronounced as MCD in photoemission reported in Ref. [22]. We introduce the dichroic spectrum $S_{D}=(I \uparrow-I \downarrow) / 2$ and the average spectrum $S_{A}=(I \uparrow$ $+I \downarrow) / 2$ to define the average MLD contrast as

$$
M_{D}=\sqrt{\frac{\int d E S_{D}^{2}(E)}{\int d E S_{A}^{2}(E)}} .
$$
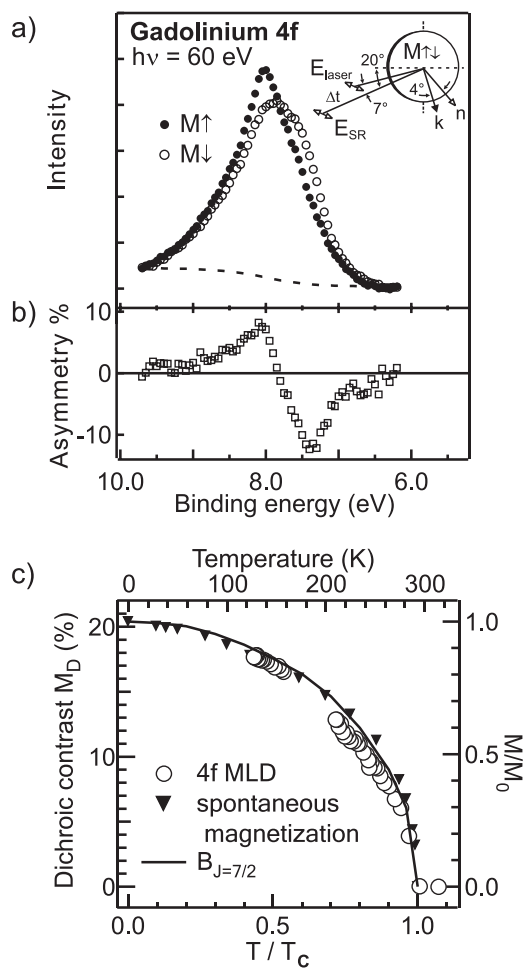

FIG. 2. (a) Gd $4 f$ core-level spectra for $\operatorname{Gd}(0001) / W(110)$. Open and filled circles correspond to opposite in-plane magnetization directions $M \uparrow$ and $M \downarrow$. The inset depicts the experimental geometry. (b) Asymmetry $(I \uparrow-I \downarrow) /(I \uparrow+I \downarrow)$ calculated from the raw data in (a). (c) Temperature dependence of the MLD contrast $M_{D}$ (open circles) compared to the reduced spontaneous magnetization $M / M(0)$ (filled triangles) and Brillouin function $B_{J=7 / 2}$ (solid line) after Ref. [14].

High-resolution photoemission spectra (not shown) allow one to resolve the $4 f$ bulk and surface components and demonstrate that for our measurement geometry the MLD is dominated by the $4 f$ bulk component $[20,23]$. As is evident from Fig. 2(c), the MLD contrast (open circles) is an appropriate signature of the bulk magnetization. It shows a temperature dependence that follows the spontaneous magnetization $M / M(0)$ (filled triangles) when aligned around $T / T_{C}=0.5$ to data from Ref. [14]. The small differences are attributed to differences in the remanent magnetization measured in MLD and the spontaneous magnetization in $\mathrm{Gd}$ single crystals extrapolated in Ref. [14] from field dependent studies. The overall temperature dependence roughly follows the Brillouin function $B_{J}$ for $J=7 / 2$. We conclude that the dichroic contrast $M_{D}$ reflects $M$ and allows us to monitor the magnetic order of the $4 f$ spin system. We assume that this likewise holds on ps time scales.

The $4 f$ core-level spectra recorded for overlapping laser pump and synchrotron probe pulses are depicted in Fig. 3(a) by filled and open symbols ( $M \uparrow$ and $M \downarrow$, laser on), $F_{a b s}=3.5 \mathrm{~mJ} / \mathrm{cm}^{2}$ [24]. Spectra for opposite magnetization direction and with or without optical excitation 
were recorded subsequently. Superimposing the unpumped spectra (solid lines, laser off) reveals that the MLD contrast is reduced upon excitation. This is emphasized in Fig. 3(b) where the dichroic spectra $S_{D}$ with and without optical excitation are compared; the dichroic contrast defined in Eq. (1) decreases by about $20 \%$ upon laser excitation. In addition, the spectra shift to higher kinetic energy and exhibit a slight broadening as illustrated in Fig. 3(c) by the $S_{A}$ spectra. The first and second moments of $S_{A}$ are calculated after subtracting a Shirley background and yield a shift of the center of mass of $135 \mathrm{meV}$ and a homogenous broadening of $0.8 \%$. These spectral changes are attributed to a transient space-charge layer generated by multiphoton photoemission of low energy electrons from the $p$-polarized pump pulse. Photoelectrons from the synchrotron pulse with their kinetic energy of about $50 \mathrm{eV}$ are thus accelerated on their way to the spectrometer.

Since the peak shift to lower binding energy and the homogenous broadening are independent of magnetization direction, the MLD contrast defined in Eq. (1) remains unaffected. As the change of dichroic contrast is much larger than the pump-induced peak broadening, third and higher order corrections are negligible [23,25]. Therefore, we can safely correct for this parasitic effect, and the data in Figs. 3(a) and 3(b) have been shifted in order to display the drop of the dichroic contrast upon optical excitation clearly.

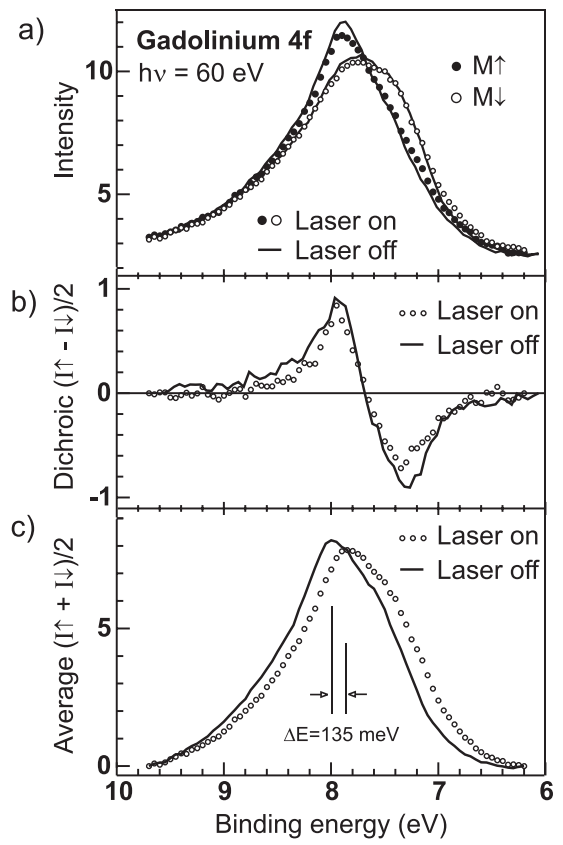

FIG. 3. (a) Gd $4 f$ core-level photoemission spectra recorded for overlapping laser pump and synchrotron probe pulses. Open and filled circles correspond to opposite in-plane magnetization directions $M \uparrow$ and $M \downarrow$. (b) Dichroic spectra $(I \uparrow-I \downarrow) / 2$ calculated from the data in (a). (c) Average spectra $(I \uparrow+I \downarrow) / 2$. The center of mass shifts by $135 \mathrm{meV}$ to lower binding energy upon laser excitation.
Finally, the pump-induced relative changes of the MLD contrast are evaluated according to

$$
\frac{\Delta M_{D}(\Delta t)}{M_{D}}=\frac{M_{D}^{\mathrm{on}}(\Delta t)-M_{D}^{\mathrm{off}}}{M_{D}^{\mathrm{off}}}=\frac{\Delta M(\Delta t)}{M} .
$$

The resulting transient magnetization is shown in Fig. 4 as a function of pump-probe delay $\Delta t$ (open circles). A $20 \%$ drop is followed by a recovery within $1 \mathrm{~ns}$. The drop in the MLD contrast demonstrates that the $4 f$ magnetic order is reduced upon optical excitation of the $5 d 6 s$ valence electrons. One may argue that this is a pure thermal effect, since we probe the $4 f$ core level with a 50 ps time resolution and electron-lattice equilibration takes $1.5 \mathrm{ps}$. A calculation of the transient magnetization from the transient lattice temperature [Fig. 1(b)] and the temperaturedependent magnetization from Fig. 2(c) gives the transient magnetization under the assumption that thermal equilibrium is established at all delays. This thermal scenario of $M(\Delta t)$ is plotted in Fig. 4 by the dashed line. As the lattice reaches a temperature close to $T_{C}$ at $1.5 \mathrm{ps}$ after the pump pulse, the magnetization drops by $80 \%$. To account for the time resolution, this temperature dependence is convoluted with a Gaussian of 50 ps full width at half maximum (FWHM) and depicted by the solid line in Fig. 4. Starting at about $80 \mathrm{ps}$, the measured $4 f$ MLD and the calculated magnetization agree nicely, which substantiates our modeling of $T_{l}(\Delta t)$. The measured drop of the magnetization at earlier times is by a factor of 2 smaller than expected from the thermal modeling (solid line), which is consistently reproduced for different $F_{a b s}$ [26]. This striking deviation of the data from the thermal estimate before 80 ps have elapsed shows directly that equilibrium between the Gd spin system and lattice is not established in this time regime. The $4 f$ spin-lattice relaxation thus takes much longer than the electron-lattice equilibration. Our result corroborates the pioneering study of Vaterlaus et al. [27], now by directly measuring the magnetic order of the $\mathrm{Gd} 4 f$ spin system. After 80 ps the recovery of the magnetization

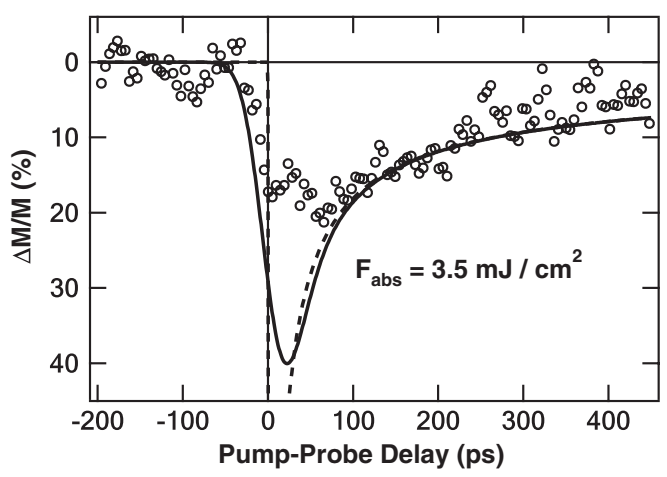

FIG. 4. Transient, relative change of the magnetization: measured $4 f$ dichroism (open circles), calculated from lattice temperature and magnetization in Figs. 1(b) and 2(c) (dashed line), and convoluted with a Gaussian of 50 ps FWHM (solid line). 
follows the cooling of the laser spot and is well described by $T_{l}(\Delta M / M \simeq 1 / e$ at $350 \pm 100 \mathrm{ps})$.

In contrast to $\mathrm{Gd}$, recovery of the ultrafast demagnetization in itinerant ferromagnets follows the evolution of $T_{l}$ once electron-phonon equilibration has been established $[1,2,6,7]$. For $\mathrm{Gd}$ this equilibration of the excited state involves more than one time scale [cf. Fig. 1(a)], because the optical excitation occurs in the valence band but the magnetic moment is dominated by localized $4 f$ electrons. Thus, beside electronic, phononic, and magnetic excitations, which affect the $\mathrm{Gd}$ valence band, equilibration includes the $4 f$ magnetic moment. Equilibrium is established, as we have shown here, on a much slower time scale of $80 \mathrm{ps}$ by spin-lattice interaction. Since valence-band electrons and the lattice cool down on this time scale, the laser-induced demagnetization in Gd is severely hampered compared to $\mathrm{Fe}, \mathrm{Co}$, and $\mathrm{Ni}$.

To summarize, we have established that the transient dichroic photoemission spectrum of the $4 f$ core level monitors the Gd magnetization dynamics. We observe a drop of the magnetization by about $20 \%$ upon laser excitation. This is by a factor of 2 smaller than expected from a purely thermal-equilibrium description based on the transient lattice temperature. We conclude that up to $80 \mathrm{ps}$ after laser excitation the $4 f$ spin system is not in equilibrium with the lattice [27]. In contrast to itinerant ferromagnets the magnetization recovers slowly, which is attributed to heat diffusion after laser excitation [18]. What is more, the experiment demonstrates the potential of time-resolved core-level photoelectron spectroscopy to study ultrafast demagnetization at surfaces. In the future ultrashort pulses of soft x-ray sources will allow us to investigate intraatomic exchange as well.

We thank the BESSY femtoslicing group, H. Dürr, T. Kachel, Ch. Stamm, N. Pontius, and M. Wietstruk for valuable discussions and the BESSY staff, in particular, R. Follath, for expert support. Financial support by the DFG through SPP 1133 is gratefully acknowledged.

*Present address: Femtolasers Production $\mathrm{GmbH}$, Fernkorngasse 10, 1100 Vienna, Austria.

'Present address: BESTEC GmbH, Carl-Scheele Straße 14, 12489 Berlin, Germany.

Present address: BESSY-GmbH, Albert-Einstein-Straße 15, 12489 Berlin, Germany.

§bovensie@physik.fu-berlin.de

"weinelt@mbi-berlin.de

[1] E. Beaurepaire, J.-C. Merle, A. Daunois, and J.-Y. Bigot, Phys. Rev. Lett. 76, 4250 (1996).

[2] J. Hohlfeld, E. Matthias, R. Knorren, and K.H. Bennemann, Phys. Rev. Lett. 78, 4861 (1997).
[3] L. Guidoni, E. Beaurepaire, and J.-Y. Bigot, Phys. Rev. Lett. 89, 017401 (2002).

[4] B. Koopmans, M. van Kampen, and W. J. M. Jonge, J. Phys. Condens. Matter 15, S723 (2003).

[5] J.-Y. Bigot, L. Guidoni, E. Beaurepaire, and P. N. Saeta, Phys. Rev. Lett. 93, 077401 (2004).

[6] M. Cinchetti et al., Phys. Rev. Lett. 97, 177201 (2006).

[7] B. Koopmans, J. J. M. Ruigrok, F. D. Longa, and W. J. M. de Jonge, Phys. Rev. Lett. 95, 267207 (2005).

[8] W. S. Fann, R. Storz, H. W. K. Tom, and J. Bokor, Phys. Rev. B 46, 13592 (1992).

[9] S. I. Anisimov, B. L. Kapeliovich, and T. L. Perel'man, Sov. Phys. JETP 39, 375 (1974).

[10] U. Bovensiepen, J. Phys. Condens. Matter 19, 083201 (2007).

[11] E. Lassner and W.-D. Schubert, Tungsten (Kluwer Academic, New York, 1999); J. G. Fujimoto, J. M. Liu, E. P. Ippen, and N. Bloembergen, Phys. Rev. Lett. 53, 1837 (1984); Handbook of Physical Quantities, edited by I. S. Grigoryev and E. Z. Meilikhov (CRC Press, Boca Raton, 1997).

[12] Y. P. Meshcheryakov and N. M. Bulgakova, Appl. Phys. A 82, 363 (2006).

[13] B. Koopmans, in Spin Dynamics in Confined Magnetic Structures II, edited by B. Hillebrands and K. Ounadjela, Topics in Applied Physics, Vol. 87 (Springer, Berlin, 2003).

[14] H. E. Nigh, S. Legvold, and F. H. Spedding, Phys. Rev. 132, 1092 (1963).

[15] R. Ahuja, S. Auluck, B. Johansson, and M. S. S. Brooks, Phys. Rev. B 50, 5147 (1994).

[16] C. S. Arnold and D. P. Pappas, Phys. Rev. Lett. 85, 5202 (2000).

[17] A. Melnikov et al., Appl. Phys. B 74, 723 (2002); Phys. Rev. Lett. 91, 227403 (2003); J. Opt. Soc. Am. B 22, 204 (2005).

[18] M. Lisowski et al., Phys. Rev. Lett. 95, 137402 (2005).

[19] A. F. Bartelt et al., Appl. Phys. Lett. 90, 162503 (2007).

[20] O. Krupin, Ph.D. thesis, Freie Universität Berlin, Germany, 2004, http://www.diss.fu-berlin.de/2004/249/.

[21] T. Gießel, D. Bröcker, P. Schmidt, and W. Widdra, Rev. Sci. Instrum. 74, 4620 (2003); D. Bröcker, T. Gießel, and W. Widdra, Chem. Phys. 299, 247 (2004).

[22] K. Starke, E. Navas, L. Baumgarten, and G. Kaindl, Phys. Rev. B 48, 1329 (1993).

[23] H. Prima-Garcia, Ph.D. thesis, Freie Universität Berlin, Germany, 2007.

[24] Average laser intensity and image of the laser focus on a fluorescence plate are measured at the sample position.

[25] Since a propagating photoelectron would be accelerated by a space-charge cloud created after the photoemission event, the constant MLD contrast we observe in Fig. 4 before excitation supports our data analysis.

[26] At $F_{a b s}=4.2 \mathrm{~mJ} / \mathrm{cm}^{2}$ the drop amounts to about $26 \%$; $F_{a b s}=3.0 \mathrm{~mJ} / \mathrm{cm}^{2}$ leads to a maximum decrease of $10 \%$.

[27] A. Vaterlaus, T. Beutler, and F. Meier, Phys. Rev. Lett. 67, 3314 (1991). 